\title{
Guanidinoneomycin-Maleimide Molecular Transporter: Synthesis, Chemistry and Cellular Uptake
}

Received 00th January 20xx, Accepted 00th January 20xx DOI: $10.1039 / x 0 x \times 00000 x$

\author{
Kaivin Hadidi, ${ }^{a}$ Maria Cristina Bellucci, ${ }^{b}$ Sergio Dall'Angelo, ${ }^{c}$ Alasdair Leeson-Payne, ${ }^{d}$ Justin J. \\ Rochford, ${ }^{d}$ Jeffery D. Esko, ${ }^{\text {e }}$ Yitzhak Tor, ${ }^{*, a}$ Alessandro Volonterio*,f
}

\begin{abstract}
Guanidinoglycosides are a class of non-cytotoxic molecular transporters capable of delivering high molecular weight bioactive cargos into cells at low nanomolar concentrations. Efficient bioconjugation with guanidinoglycosides has been previously demonstrated by utilizing a guanidinoneomycin decorated with a reactive but also unstable $\mathrm{N}$ hydroxysuccinimmide ester-containing linker. Herein we report the synthesis, chemistry, and application of a new, stable guanidinoneomycin derivative armed with a highly specific maleimide moiety which allows for thiol-maleimide click chemistry, a highly popular bioconjugation strategy, widening the field of application of these intriguing and useful delivery vehicles.
\end{abstract}

\section{Introduction}

Poor cell membrane permeability is a key limiting factor in the discovery and the development of potential therapeutic agents designed to modulate intracellular components. ${ }^{1-3}$ To overcome this drawback, different chemical strategies have been developed, depending on the nature of the bioactive molecule. For instance, small hydrophilic molecular drugs are converted into pro-drugs for enhanced membrane permeability, ${ }^{4}$ while the physicochemical features of larger biomolecules, such as peptides or proteins, have been modified through backbone cyclization or side chain "stapling", amide bond masking, or dressing with guanidine groups. ${ }^{4-7}$ Alternatively, a common strategy yet also the most exploited for helping internalization of both small molecules and high molecular weight biomacromolecules relies on the use of molecular transporters encompassing antibodies, dendrimers, biocompatible polymer nanocarriers, liposomes, and cell penetrating peptides (CPPs), among others. ${ }^{8-12}$

CPPs have played a pivotal role since the discovery of the 11 mer Tat-peptide and its role in the transduction of HIV into cells. ${ }^{13,14}$ Despite the extensive work done, which led to the development of various arginine rich CPPs, including cyclic, ${ }^{15}$

\footnotetext{
a. Department of Chemistry and Biochemistry, University of California, San Diego, 9500 Gilman Drive, La Jolla, California 92093, United States.

b. Department of Food, Environmental and Nutritional Sciences, Università degli Studi di Milano, via Celoria 2, 20133 Milano, Italy.

c. Institute of Medical Sciences, University of Aberdeen, AB25 2ZD Aberdeen, U.K.

d. The Rowett Institute and Aberdeen Cardiovascular and Diabetes Centre,

University of Aberdeen, Aberdeen AB25 2ZD, UK.

e. Department of Cellular and Molecular Medicine, University of California, San Diego, 9500 Gilman Drive, La Jolla, California 92093, United States.

f. Department of Chemistry, Material and Chemical Engineer "Giulio Natta", Politecnico di Milano, via Mancinelli 7, 20131 Milano, Italy

Electronic Supplementary Information (ESI) available: [details of any supplementary information available should be included here]. See DOI: 10.1039/x0xx00000x
}

non-peptidic, ${ }^{16}$ and stimuli-responsive CPPs, ${ }^{17}$ the "ideal" CPP able to overcome the drawbacks associated with their use, such as nonspecificity, susceptibility to proteolytic degradation, cytotoxicity, and high production costs, is still missing. ${ }^{18,19}$ Nonetheless, these studies have provided insight for the design of new molecular transporters, such as the significance of multiple guanidinium groups that interact with cell surface proteoglycans through bidentate hydrogen bonds. ${ }^{20}$ Since this early observation, different guanidinium-rich molecular transporters have been successfully developed, encompassing inositol, ${ }^{21}$ carbohydrate scaffolds, ${ }^{22,23}$ dendrimers, ${ }^{24}$ carbon nanotubes, ${ }^{25}$ calix[n]arenes, ${ }^{26,27}$ and bicyclic guanidinium tetramers. ${ }^{28}$

Guanidinoglicosides, namely aminoglycoside antibiotics substituting the ammonium groups for guanidinium groups, have been introduced by some of us as non-cytotoxic, highly efficient molecular transporters. ${ }^{29}$ In particular, guanidinonemycin (GNeo) showed higher cellular uptake compared to $\mathrm{Arg}_{9} \mathrm{CPP}$, being able to translocate high molecular weight cargos into cellular lysosomes at low nanomolar carrier concentrations through a heparan sulfate-dependent pathway. ${ }^{30-33}$ To facilitate conjugation to biomolecules, GNeo has been decorated with a linker bearing a highly reactive $\mathrm{N}$ hydroxysuccinimmide ester (GNeo-NHS, Figure 1). ${ }^{34}$ GNeo-NHS of first and second generation derivatives have been successfully used to functionalize two lysosomal enzymes, $\beta$-Dglucoronidase (GUS) and $\alpha$-L-iduronidase (IDUA), exploiting accessible lysine residues. The corresponding functional conjugates were efficiently delivered into cells lacking these lysosomal enzymes. ${ }^{34}$ Moreover, due to increased uptake into neurons and astrocytes, GNeo-IDUA conjugates has been shown to reduce glycosaminoglycan storage and neuropathological hallmarks of disease in the olfactory bulb and cerebral cortex by repetitive intranasal administrations in Mucopolysaccharidosis I mice deficient in IDUA. ${ }^{35}$ 
Although GNeo conjugation to biomolecules using GNeo-NHS occurs efficiently, this approach suffers from certain limitations: 1) lysine residues exposed on the surface of the macromolecule are needed, 2) other accessible nucleophilic moieties, such as the thiol of Cys or the hydroxy group of Tyr, may somewhat compromise selectivity, 3) conjugation takes place through a covalent, irreversible linkage, potentially affecting bioactivity, and 4) GNeo-NHS is susceptible to hydrolysis, which renders its synthesis, purification and storage quite demanding. To overcome some of these drawbacks, liposomes decorated with GNeo (GNeosomes) ${ }^{36,37}$ as well as PAMAM dendrimers ${ }^{38-40}$ and calix[n]arenes have been recently developed. ${ }^{41}$

One of the most exploited techniques for bioconjugation is the conjugate addition of thiols to maleimides. ${ }^{42,43}$ This "click" reaction occurs rapidly in aqueous buffer solutions at $\mathrm{pH} 7.4$, typically in high yields with good selectively for thiols with limited side-products, leading to the formation of a stable thiolmaleimide adduct. ${ }^{44}$ Indeed, site-selective cysteine modification in proteins is probably the most popular choice for protein bioconjugation as evidenced by its use in the preparation of antibody-drug conjugates. ${ }^{45}$

We report herein the synthesis of a new guanidinoneomycin molecular transporter bearing a maleimide moiety (GNeo-Mal 1a, Figure 1). We demonstrated that GNeo-Mal reacts efficiently with cysteine-modified biomolecules to promote cellular uptake, widening the versatility and potential of this class of fascinating, non-peptidic molecular transporters.
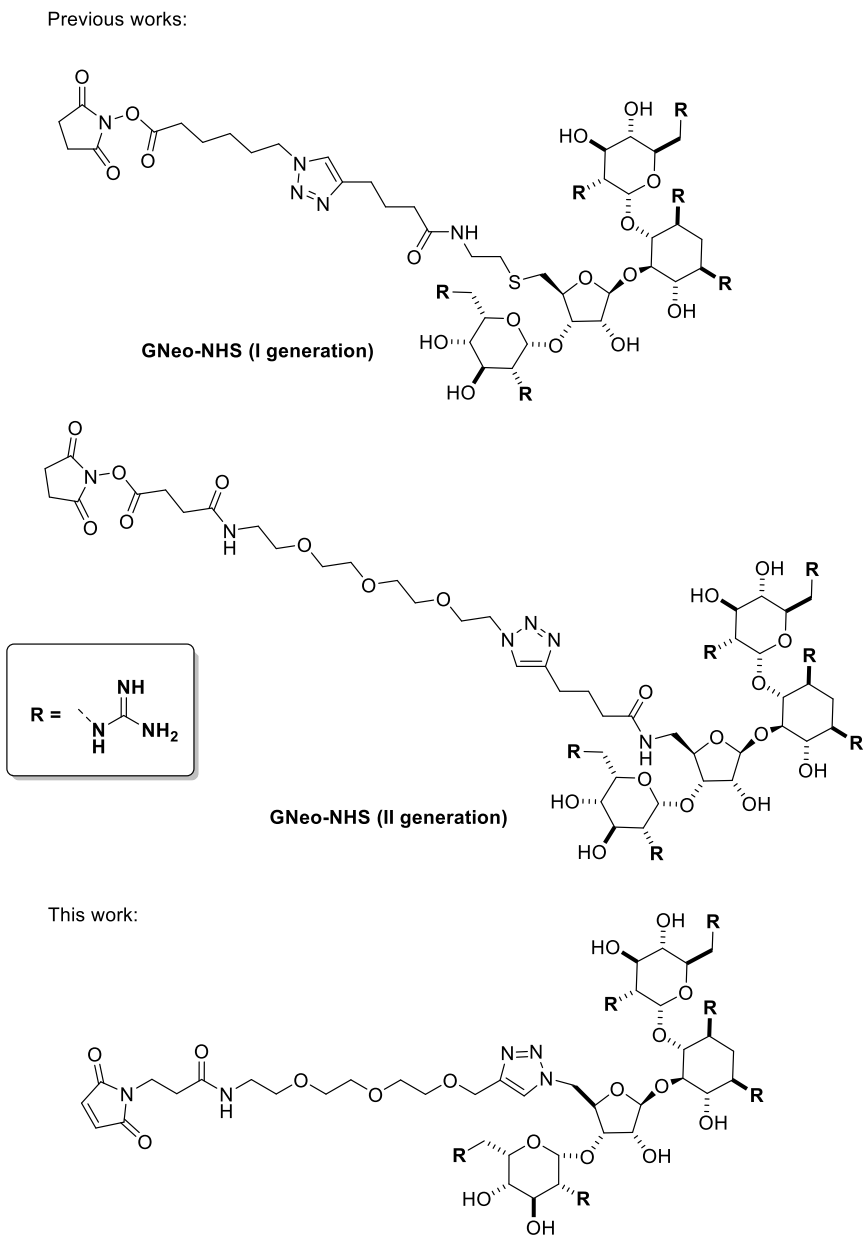

GNeo-Mal 1a

Figure 1. Structures of GNeo-NHSs and GNeo-Mal

\section{Results and discussion}

\section{Synthesis and chemistry of GNeo-Mal.}

Bioconjugation is an important technique which tethers an exogeneous moiety to a biomolecule to endow the latter with beneficial properties. ${ }^{46}$ The reaction between NHS-activated esters and amines can be considered the gold standard of protein conjugation. Accordingly, this strategy was originally utilized to attach $\mathrm{GNeo}$ molecular transporter to lysosomal enzymes GUS and IDUA to increase their cellular uptake. ${ }^{34}$ The presence of different $\varepsilon$-amino groups on solvent-exposed lysine residues of these enzymes, produced GNeo-enzyme conjugates able to efficiently reach the lysosome and affect biological activity in vitro and in vivo. ${ }^{34,35}$ Due to the instability of NHSester group in aqueous solution, the use of a large excess (from 50 to 100 molar excess ratio) of GNeo-NHS reagents was, however, necessary. Moreover, the efficiency and selectivity of bioconjugation with GNeo-NHS depend on the specific biomolecule since NHS-esters can react with other amino acid side chains of cysteine, tyrosine, serine, and threonine. These observations, along with the difficulties concerning the 
synthesis, isolation and storage of GNeo-NHS, could limit the application of this powerful non-peptidic molecular transporter. To overcome these limitations and expand the conjugation portfolio of these compounds, we synthetized GNeo-Mal 1a from $\mathrm{GNeo}^{-\mathrm{N}_{3}} \mathbf{6}$ and the ad hoc synthetized maleimidecontaining linker $\mathbf{5}$ (Scheme 1). Accordingly, maleic anhydride $\mathbf{2}$ was reacted with $\beta$-alanine affording maleimide carboxylic acid $3^{47}$ which was coupled with PEG-linker 4, prepared as previously reported in literature, ${ }^{48}$ bearing the maleimide moiety on one end and a propargyl group on the other end, leading to the formation of maleimide-alkyne PEG-linker $\mathbf{5}$. Thanks to the stability of the maleimide moiety, we could couple Boc-GNeo- $\mathrm{N}_{3} 6 \mathrm{a}$, which was prepared according to the procedure reported in literature, ${ }^{39}$ and $\mathbf{5}$ following a "standard" click protocol, namely using $\mathrm{CuSO}_{4}$ and $\mathrm{Na}$-ascorbate in the presence of catalytic TBTA in a mixture of aprotic-protic solvents (DCM/water). Full Boc-deprotection and HPLC purification yielded the target GNeo-Mal 1a as an easy to handle, stable to storage fluffy white solid. Following the same synthetic strategy, the corresponding neomycin derivative NeoMal 1b was prepared starting from Boc-Neo- $\mathrm{N}_{3}{ }^{39} \mathbf{6 b}$ to highlight the effect of guanidinylation on the cellular uptake properties of the glycoside (Scheme 1).

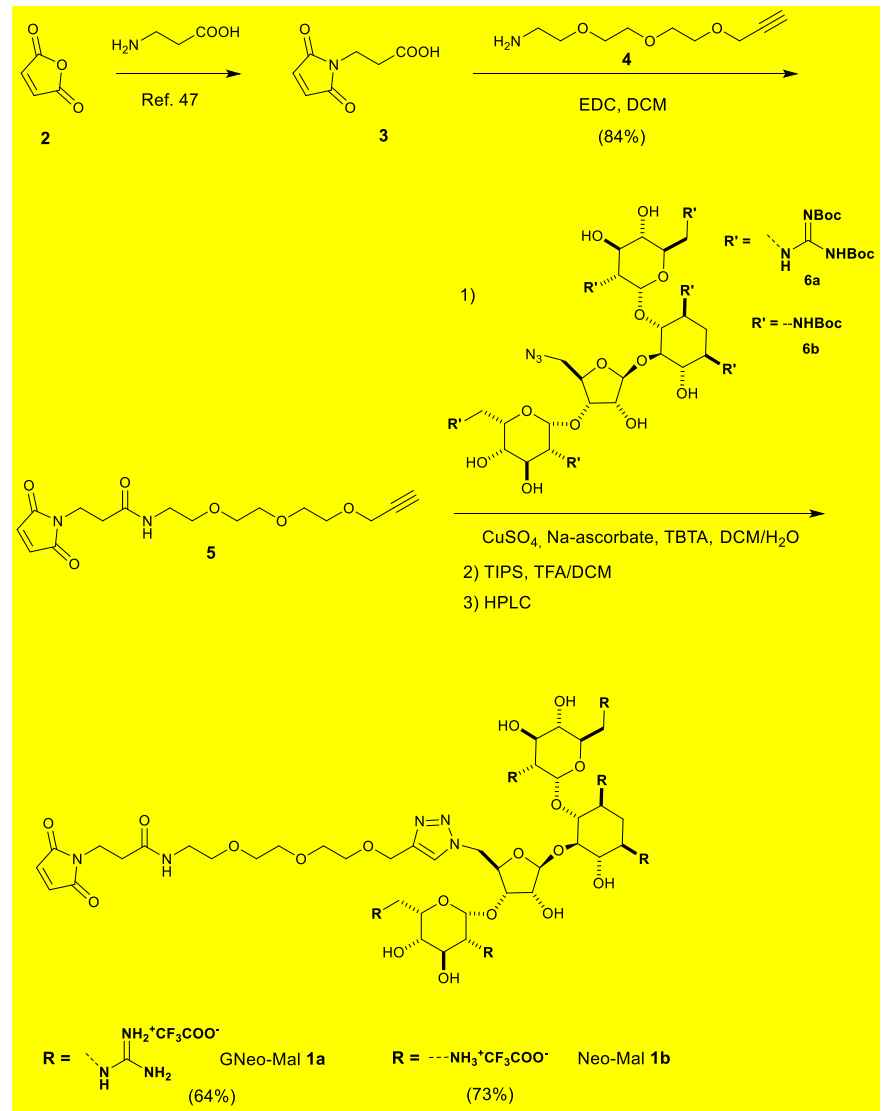

Scheme 1. Synthesis of GNeo-Mal 1a and Neo-Mal 1b.

The reactivity of (G)Neo-Mal $\mathbf{1} \mathbf{a}, \mathbf{b}$ and the capacity to deliver high molecular weight cargos into cells was demonstrated by conjugation with biotinylated cysteine (biotin-Cys) followed by a second conjugation with fluorescently labeled streptavidin- phycoerythrin (ST-PECy5, $300 \mathrm{kDa}$ ) and subsequent cellular delivery. Accordingly, we prepared biotin-Cys conjugate $\mathbf{1 0}$ according to the pathway depicted in Scheme 2. Biotin $\mathbf{7}$ was coupled with $\mathrm{N}$-Boc-ethylenediamine to obtain intermediate $\mathbf{8}^{49}$ which was Boc-deprotected and coupled with commercially available $\mathrm{N}$-acetyl-S-trityl-L-cysteine leading to the formation of thiol-free biotin-Cys $\mathbf{1 0}$ after final removal of the trityl group.

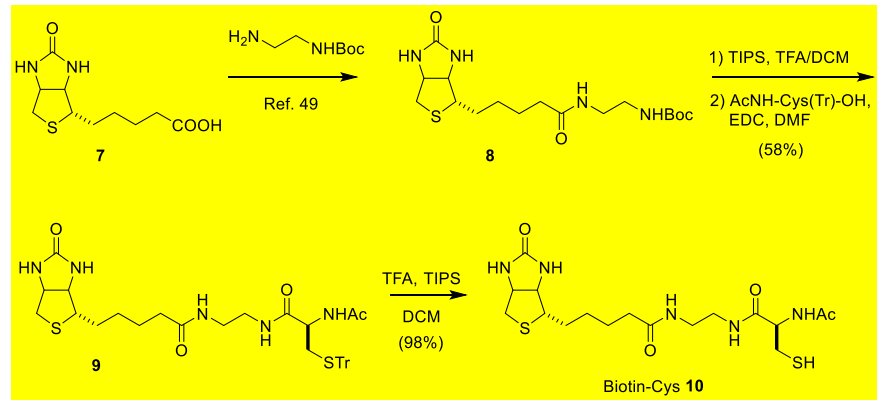

Scheme 2. Synthesis of biotin-Cys conjugate $\mathbf{1 0}$

Biotin-Cys 10 reacted smoothly with (G)Neo-Mal derivatives $\mathbf{1} \mathbf{a}, \mathbf{b}$ in less than five minutes in buffered aqueous solution at $\mathrm{pH} 7.4$ efficiently producing conjugates $\mathbf{1 1 a}$,b (Scheme 3), as evidenced by HPLC and HRMS spectroscopy (Schemes S1 and S2 in Supporting Information). Since the robustness of the thiolmaleimide linkage has been recently questioned, ${ }^{44}$ we checked the stability of GNeo-Mal-biotin conjugate 11a by dissolving it in an aqueous buffer solution at $\mathrm{pH} 7.4,37^{\circ} \mathrm{C}$ and monitoring potential degradation by HPLC over time. After 90 minutes we detected the appearance of a new peak with increased area as a function of time, showing a conversion of around $50 \%$ after 72 hours (Scheme S3 in Supporting Information). Interestingly, the new peak had a different retention time from GNeo-Mal 1a meaning that the thioether linkage in 11a was not reversible. To our delight, as evidenced by HRMS, the new product is the result of the hydrolytic ring opening of maleimide-thiol adduct which renders the thioether linkage even more stable. ${ }^{50}$ 

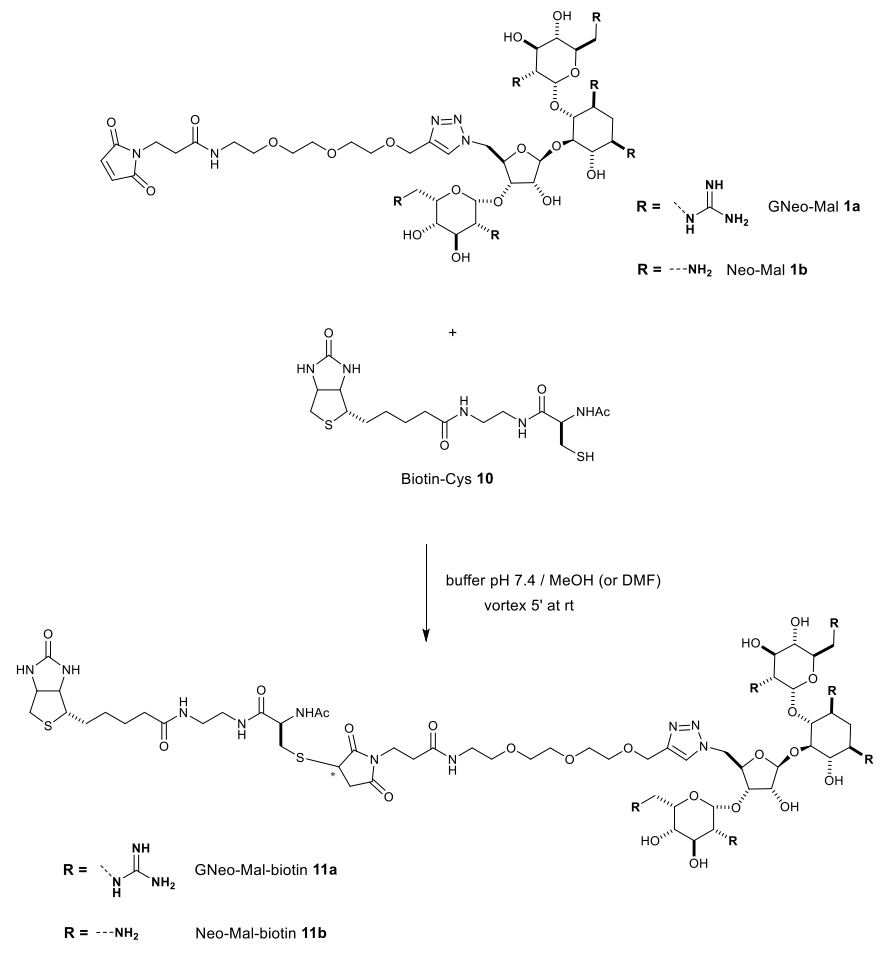

Scheme 3. Synthesis of (G)Neo-Mal-biotin conjugates 11a,b

\section{Cellular uptake of (G)Neo-Mal conjugates}

To evaluate the cellular uptake of the novel GNeo derivatives, compounds 1a, 11a, and 11b were treated with ST-PECy5 to produce tetrameric GNeo-maleimide-streptavidin/biotin complexes. Wild type Chinese hamster ovary (CHO-K1) cells were incubated with these complexes at different $\mathrm{nM}$ concentrations and the mean fluorescence intensities (MFIs) were determined and analyzed by flow cytometry (Figure 2a). As hypothesized, only the complexes that possessed both the GNeo transporter and the biotin handle (GNeo-Mal-biotin 11a) exhibited drastically increased internalized fluorescence several orders of magnitude higher than negative controls. The neomycin analogues 11b also showed a mild increase in internalization, but the effect was miniscule compared to the guanidino-modified molecular transporter 11a. Additionally, the uptake experiment in wild-type $\mathrm{CHO}-\mathrm{K} 1$ cells was repeated in the absence of albumin and thiol-rich FBS during the crucial incubation step of the streptavidin complexes resulting in a slight decrease or comparable MFls at lower nM concentrations, and an increase of internalized fluorescence at $25 \mathrm{nM}$ concentration (Figure 2b). These results remain consistent with the observation that cellular starvation attenuates macropinocytosis as the cell scrambles to obtain nutrients, rather than an irreversible exchange due to the presence of excess of thiols. ${ }^{51,52}$ Moreover, no thiol exchange was observed when conjugate 11a was incubated with excess of glutathione at $\mathrm{pH}$ 7.4.

Previously published derivatives of guanidinoneomycin molecular transporters rely on heparan sulfate proteoglycans on the cell surface for endocytosis. ${ }^{31}$ To verify GNeo-Mal 11a shares a similar mechanism, mutant pgsA745 cells, devoid of heparan sulfate, were incubated with the fluorescently labelled Streptavidin complexes (Figure 2c). Flow cytometry analysis indicated a significant drop of internalized fluorescence, cementing the necessity of heparan sulfate for endocytosis of GNeo-Mal complexes.

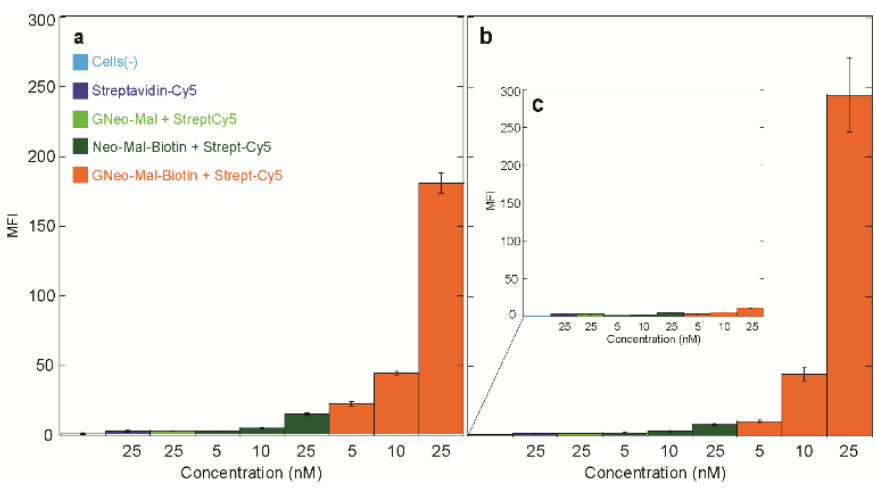

Figure 2. Cellular uptake. Cells were incubated with the carriers complexed to fluorescently labeled streptavidin (ST-PECy5) for $1 \mathrm{~h}$ at $37{ }^{\circ} \mathrm{C}$. MFI was measured by flow cytometry. The background signal from untreated cells was subtracted. A) Wildtype CHO-K1 cells incubated with ST-PECy5 (25 nM) and STPECy5 complexed to GNeo-Mal (25 nM) and (G)Neo-Mal-biotin $(5,10$, and $25 \mathrm{nM})$ as indicated. B) Wild-type CHO-K1 cells incubated without the presence of albumin and thiol-rich FBS. C) Mutant pgsA-745 cells incubated with the same conjugates. Error bars each represent the standard deviation from an average of three experiments, each of them at least in triplicate.

\section{Conjugation with SP012 peptide and cellular uptake}

To examine the effectiveness of the GNeo-Mal transporter in an additional biological setting, GNeo-Mal 1a was conjugated to a synthetic peptide SP012 (sequence GNSALHVASQHG). SP012 is a peptide designed to inhibit the function of $\gamma$-synuclein (SNCG), a protein associated with proliferation in tumour cells. ${ }^{53}$ SNCG is involved in the machinery of cellular proliferation by interacting with the mitotic checkpoint protein BUB1-related protein 1 (BubR1) which regulates spindle assembly. ${ }^{54-57}$ SP012 was designed to disrupt SNCG binding to BubR1 and consequently increase the inhibitory action of the spindle assembly checkpoint, eventually leading to a significant decrease of the cellular proliferation in metastatic lesions.

To determine whether GNeo molecular transporter would improve the delivery of SP012 peptide into cells compared to transactivating transcriptional activator transporter (Tat, sequence GYGRKKRRQRRR) which was used in the previous study, ${ }^{53}$ a cysteine decorated SP012 peptide was conjugated to GNeo-Mal 1a. Peptides were synthesized by solid phase microwave assisted peptide synthesis (SPPS) using a Liberty Blue $^{\mathrm{TM}}$ Automated Microwave Peptide Synthesizer starting from a Rink amide MBHA LL resin and exploiting a CarboMax strategy (see Supporting Information for details). To conjugate the 
GNeo-Mal 1a to SP012, an Ac-Gly-Cys-Gly motif was added at the $\mathrm{N}$-terminus of the peptide during the automated SPPS leading to peptide $\mathbf{1 2}$ (sequence GCGGNSALHVASQHG, Scheme 4). The Ac-Gly-Cys-Gly motif was selected to space the cysteine reactive site from the peptide and at the same time to mimic a non-terminal cysteine. Any attempt to conjugate GNeo-Mal 1a to the resin-bound peptide $\mathbf{1 2}$ in solid phase failed, either giving low yields or a mixture of products that proved difficult to separate, so the click step was performed in solution. Cleavage of the peptide from the resin and removal of protecting groups was performed by treating the resin with a cleavage solution of 92.5\% trifluoroacetic acid (TFA), $2.5 \%$ 1,2-ethanedithiol, $2.5 \%$ triisopropylsilane (TIPS) and 2.5\% of water leading to peptide $\mathbf{1 2}$ which, without any further purification, was conjugated with GNeo-Mal 1a in water for $24 \mathrm{~h}$ to obtain the GNeo-SP012 peptide 13 (Scheme 4 and S4. The reaction was much slower compared to the conjugation with biontin-Cys 10 probably due to steric hindrance). Analogously, Tat-SP012 14 (sequence GYGRKKRRQRRRGNSALHVASQHG) was prepared by automated SPPS and treated with a 95\% trifluoroacetic acid (TFA), $2.5 \%$ triisopropylsilane (TIS) and $2.5 \%$ of water mixture for resin cleavage and side chains deprotection. The crude peptides were purified by semi-preparative RP-HPLC.

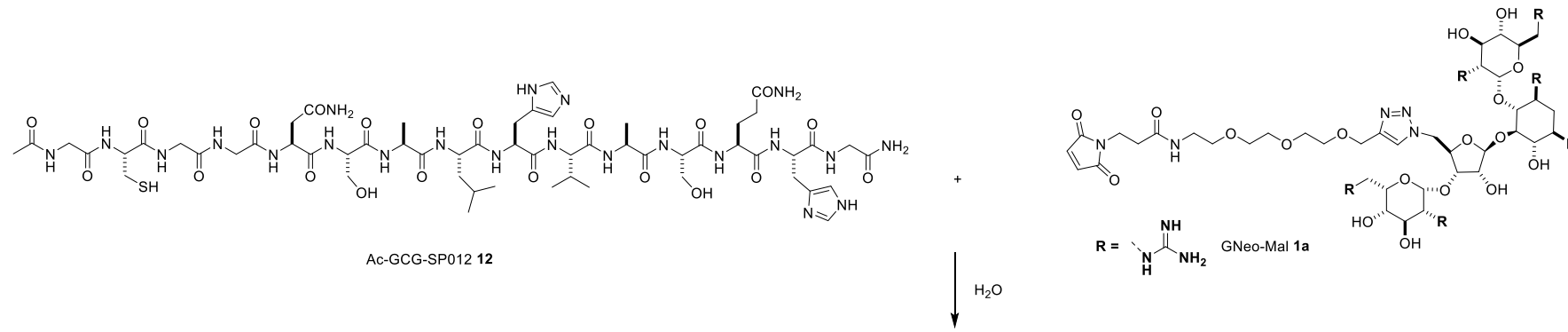

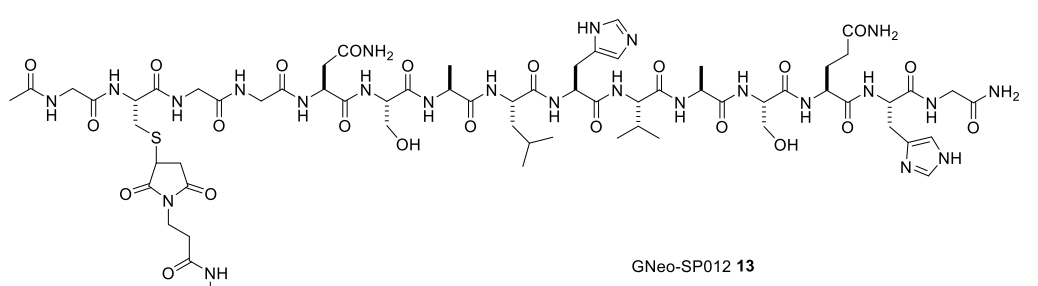

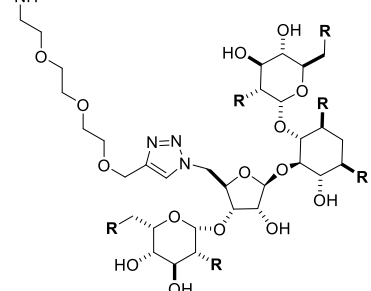

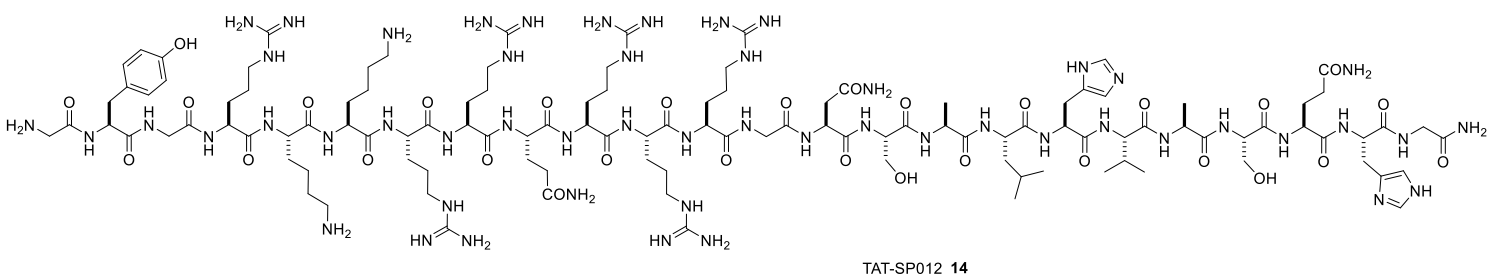

Scheme 4. Synthesis of GNeo-SP012 peptide 13 and structure of Tat-SP012 14

Proliferating Neuro2A cells were treated with either GNeoSP012 13 or TAT-SP012 14 to compare the rate of proliferation following 24 hours incubation. Treatment with the same concentration $(50 \mu \mathrm{M})$ of GNeo-SP012 13 or Tat-SP012 14 showed that the rate of proliferation was significantly reduced with GNeo-SP012 13 compared to Tat-SP012 14 (Figure 3, A). This indicates that the GNeo-SP012 13 conjugate is more effective at transporting the SP012 peptide. To ensure this was not due to toxicity of the conjugate, cellular toxicity was measured in a non-proliferating cell line. Day 8 differentiated 3T3-L1 adipocytes were treated with up to $100 \mu \mathrm{M}$ GNeo-SP012 13 or Tat-SP012 14 and cellular toxicity was measured by resazurin assay. No significant effect on toxicity was observed with either conjugate demonstrating that neither Tat-SP012 14 nor GNeo-SP012 13 resulted in cell toxicity or cell death (Figure 3, B, C). These results demonstrate that GNeo-SP012 13 
significantly reduced the rate of proliferation in Neuro2A cells and indicates that the GNeo transporter improves the cellular delivery of synthetic peptide SP012 directly compared to Tat. This demonstrates that GNeo-SP012 13 conjugate improved the delivery of the synthetic peptide SP012 in vitro compared to the Tat-SP012 conjugate 14 .
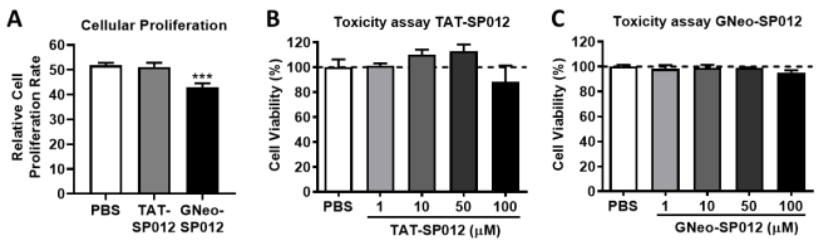

Figure 3. Effect of SP012 conjugate on cellular rate of proliferation and toxicity. (A) Cellular proliferation was measured by resazurin assay in Neuro2A cells. Neuro2A cells were maintained at sub-confluency and treated with 50 $\mu \mathrm{M}$ TAT-

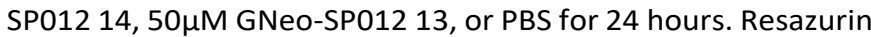
assay was performed prior to and following peptide treatment to determine effect on proliferation. (ANOVA, $n=8$, $* * * p \leq 0.001$ ). Cellular toxicity of (B) TAT-SP012 14 and (C) GNeoSP012 13 was measured by resazurin assay prior to and following treatment with of conjugates in un-proliferating differentiated adipocytes. 3T3-L1 cells were differentiated for 8 days before being treated with TAT-SP012 14, GNeo-SP012 13, or PBS control for $24 \mathrm{~h}$. (ANOVA, $\mathrm{n}=4$ ).

\section{Conclusions}

A new GNeo-based molecular transporter has been developed by anchoring a maleimide-bearing linker to the GNeo scaffold. The resulting GNeo-Mal derivative, obtained in good overall yield, is stable, easy to handle and can be to recovered by HPLC. Since it can be stored, it provides an attractive route for bioconjugation through thiol-maleimide click chemistry. We have demonstrated that GNeo-Mal reacts in a straightforward manner with small molecules, such as a biotin-Cys conjugate, and with a Cys-decorated 15-mer oligopeptide, leading to the formation of conjugates that were able to promote the cellular uptake of $300 \mathrm{kDa}$ fluorescently labeled streptavidin and SP012 peptide, respectively. These results widen the potential and utility of this highly efficient, non-cytotoxic molecular transporter, and provides an alternative for less efficient (and potentially cytotoxic) cell penetrating peptides. Since thiolmaleimide click chemistry is a commonly utilized strategy in the bioconjugation toolbox for not only peptides and proteins, but possibly any bio(macro)molecule due to the different strategies reported in the literature to introduce a sulfhydryl reacting group, ${ }^{58-60}$ we believe that the GNeo-Mal molecular transporter could find wide applications in the development of novel drugs by improving cellular uptake of therapeutic entities.

\section{Experimental Section}

Materials. Materials obtained from commercial suppliers were used without further purification. Chemical and reagents were obtained from Sigma Aldrich. Fmoc protected amino acids were obtained from CEM, DMF and HPLC grade water and Acetonitrile were obtained from VWR UK. (G)Neo- $\mathrm{N}_{3} \mathbf{6 a}, \mathbf{b}^{39}$ and compounds $3^{47}, \mathbf{4}^{48}$ and $\mathbf{8}^{49}$ were prepared as reported in literature. PBS, FBS, F-12 nutrient mixture (HAM) and streptavidin-Cy5 were purchased from Life Technologies (San Diego, CA). Trypsin-EDTA was purchased from VWR (Mediatech, Manassas, VA USA). All cellular uptake experiments were carried out in Corning 24-well plates.

Instrumentation. NMR were recorded on either a Varian Mercury $400 \mathrm{MHz}$ or $500 \mathrm{MHz}$ spectrometers. Mass spectra were recorded at the UCSD Chemistry and Biochemistry Mass Spectrometry Facility; low resolution mass spectrometry (LR-MS) analysis was performed on a Thermo LCQdeca mass spectrometer using electrospray ionization (ESI) as the ion source. An Agilent 6230 time of flight mass spectrometer (TOFMS) was employed for high resolution MS (HRMS) analysis using ESI as the ion source. Reversed phase HPLC purification of (G)Neo-Mal (CLIPEUS, C18, $5 \mu \mathrm{m}, 10 \times 250 \mathrm{~mm}$, Higgins analytical) and analysis (Eclipse, XDB-C18, $5 \mu \mathrm{m}, 4.6 \times 150 \mathrm{~mm}$ ) were carried out on an Agilent 1200 series instrument. Reversed phase HPLC purification of peptides were carried out on an Agilent 1260 series Preparative instrument (Phenomenx Jupiter column 21.6x250 $\mathrm{mm}, 300 \AA, 5 \mu \mathrm{m}$, ) and the reversed phase HPLC analysis of the peptide (Phenomenx Jupiter column $21.6 \times 250 \mathrm{~mm}, 300 \AA$, $5 \mu \mathrm{m}$,) Peptides were prepared through 9-Fluorenylmethoxycarbonyl (Fmoc) solid phase microwave assisted peptide synthesis (SPPS) using a Liberty Blue ${ }^{\mathrm{TM}}$ Automated Microwave Peptide Synthesizer (CEM). Coupling agents used: $1 \mathrm{M} \mathrm{N}, \mathrm{N}^{\prime}$-diisopropylcarbodiimide (DIC) and $1 \mathrm{M}$ ethyl cyano(hydroxyimino)acetate (Oxyma pure) solution in dimethylformamide as additive. Fmoc deprotection was performed with a $20 \%$ piperidine solution in DMF.

Cell Culture. All cell lines were grown as previously reported. ${ }^{36}$

Chemical Synthesis. The synthetic procedures and characterizations of all new compounds and peptides are described in the Supporting Information.

Cellular Uptake. Wild-type CHOK1 and mutant pgsA-745 cells were seeded onto a 24-well plate at a density of 100,000 cells per well and grown to $80 \%$ confluency overnight. The modified amino and guanidinoglycosides were then bound to a Cy5-labelled streptavidin to form tetravalent biotin-streptavidin conjugates by incubating the compounds with streptavidin-Cy5 (5:1) in a MilliQ:PBS (1:1) solution while protected from light for 20 minutes. The streptavidin conjugates were diluted to the desired concentrations in $\mathrm{F}-12$ growth medium containing 10\% FBS (no FBS added for serum-free experiment). The cells were washed with PBS and incubated with 300 $\mu \mathrm{L}$ of the fluorescent carrier solutions for $1 \mathrm{~h}$ at $37^{\circ} \mathrm{C}$ in a $5 \% \mathrm{CO} 2$ atmosphere. The cells were then washed twice with $300 \mu \mathrm{L}$ of PBS and detached with $60 \mu \mathrm{L}$ of trypsin-EDTA for 10 minutes, followed by a dilution with $0.1 \%$ BSA in PBS, and analyzed by FACS. 
Cellular Proliferation and Toxicity. Cellular 3T3-L1 cells were maintained in culture using 3T3-L1 Growth Media (DMEM GlutaMax (GIBCO, \#D5671), 10\% (v/v) New-born Calf Serum (Sigma, \#N4762), 2\% (v/v) Streptomycin/Penicillin/Glutamine (GIBCO, \#10376-016), $1 \%(\mathrm{v} / \mathrm{v}$ ) Sodium Pyruvate solution (Sigma, \#S8636), and 1\% (v/v) Non-Essential Amino Acid solution (Sigma, \#M7145)). To stimulate differentiation of 3T3-L1 pre-adipocytes into mature adipocytes, cells were cultured in 3T3-L1 Differentiation Media (DMEM GlutaMax (GIBCO, \#D5671), 10\% (v/v) Foetal Bovine Serum (GIBCO, \#10500-064), 2\% (v/v) Streptomycin/Penicillin/Glutamine (GIBCO, \#10376-016), 1\% (v/v) Sodium Pyruvate solution (Sigma, \#S8636), and $1 \%(\mathrm{v} / \mathrm{v}$ ) Non-Essential Amino Acid solution (Sigma, \#M7145)). For the first two days of differentiation (day 0- day 2) 3T3-L1 Differentiation Media was supplemented with $0.85 \mu \mathrm{M}$ Insulin

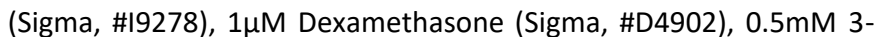
isobutyl-1-methylxanthine (IBMX) (Sigma, \#I5879), and $2 \mu \mathrm{M}$ Rosiglitazone (Sigma, \#R2408). From day 2 to day 4 of differentiation, 3T3-L1 Differentiation Media was supplemented with $0.85 \mu \mathrm{M}$ Insulin (Sigma, \#19278). Neuro2A cells were maintained in culture in Growth Media (DMEM GlutaMax (GIBCO, \#D5671) with 10\% (v/v) New-born Calf Serum (Sigma, \#N4762). Cell toxicity and cell proliferation was measured using resazurin cell viability assay (R\&D Systems, \#AR002). For all determinations of cell viability, measurements were taken prior to experimental treatment and following to ensure any preexisting variation in viability was eliminated. Cells were incubated in media with $10 \%(\mathrm{v} / \mathrm{v})$ resazurin and readings were taken according to the manufacturers protocol at multiple time points on a SpectraMAX 190 spectrophotometer (Molecular Devices, USA) These readings were normalised to the initial pre-experimental measurements.

\section{Author Contributions}

AV planned the chemical synthesis. AV and $\mathrm{MCB}$ carried out all chemical reactions, and isolated, purified and analysed all reaction products. KH and ALP conducted and evaluated the biological assays. SD carried out the peptide-conjugates synthesis. YT supervised the chemical part and revised the manuscript. JE and JR supervised the biological studies. AV, KH, ALP and SD wrote the manuscript.

\section{Conflicts of interest}

There are no conflicts to declare.

\section{Acknowledgements}

We thank the Fulbright Program for a Research Scholar Fellowship (A.V.) and the National Science Foundation for support (via grant number $\mathrm{CHE}-1303554)$, the Biotechnology and Biological Sciences Research Council [BB/K017772/1] (J.J.R.) as well as the Chemistry and Biochemistry MS and NMR Facilities.

\section{Notes and references}

1 R. Zhang, X. Qin, F. Kong, P. Chen, G. Pan, Drug Del. 2019, 28, 328.

2 P. Matsson, B. C. Doak, B. Over, J. Kihlberg, Adv. Drug Del. Rev. 2016, 101, 42.
3 J. Y. Groves, Dev. Cell 2019, 48, 15-16.

4 J. Rautio, H. Kumpulainen, T. Heimbach, R. Oliyai, D. Oh, T. Järvinen, J. Savolainen Nat Rev Drug Discov. 2008, 7, 255.

5 E. M. Driggers, S. P. Hale, J. Lee, N. K. Terrett Nat. Rev. Drug Discov. 2008, 7, 608-624.

6 Q. Chu, R. E. Moellering, G. J. Hilinski, Y.-W. Kim, T. N. Grossman, J. T. Yeh, G. L. Verdine Med Chem Commun. 2015, 6, 111.

7 G. H. Bird, E. Mazzola, K. Opoku-Nsiah, M. A. Lammert, M. Godes, D. S. Neuberg, L. D. Walensky Nat. Chem. Biol. 2016, 12, 845 .

8 R. V. J. Chari ACS Med. Chem Lett. 2016, 11, 974

9 H. I. Chang, M. K. Yeh Int. J. Nanomed. 2012, 7, 49.

10 M. Slingerland, H. J. Guchellar, H. Gelderblom Drug Discovery Today 2012, 17, 160.

11 W. Chen, S. Zhou, L. Ge, W. Wu, X. Jiang Biomacromolecules 2018, 19, 1732-1745.

12 L. Gui, X.-H. Zhang, Z.-Y. Qiao, H. Wang ChemNanoMat 2020, 6, 1.

13 A. D. Frankel, C. O. Pabo Cell 1988, 55, 1189.

14 M. Green, P. M. Loewenstein Cell 1988, 55, 1179.

15 S. E. Park, M. I. Sajid, K. Parang, R. K. Tiwari Mol. Pharmaceutics 2019, 16, 3727.

16 E. G. Stanzl, B. M. Trantow, J. R. Vargas, P. A. Wender Acc. Chem. Res. 2013, 46, 2944.

17 F. Perche Process, 2019, 7, 727.

18 J. Habault, J.-L. Poyet Molecules 2019, 24, 927.

19 F. Nadal-Bufi, S. T. Henriques Pept. Sci. 2020, 112, 1.

20 E. Wexselblatt, J. D. Esko, Y. Tor J. Org. Chem. 2014, 79, 6766.

21 K. M. Maiti, O.-Y. Jeon, W. S. Lee, D.-C. Kim, K.-T. Kim, T. Takeuchi, S. Futaki, S.-K. Chung Angew. Chem. Int. Ed. 2006, 45, 2907.

22 G. Biswas, O.-Y. Jeon, W. S. Lee, D.-C. Kim, K.-T. Kim, S. Lee, S. Chang, S.-K. Chung Chem. Eur. J. 2008, 14, 9161.

23 J. Im, S. Kim, Y.-H. Jeong, W. Kim, D. Lee, W. S. Lee, Y.-T. Chang, K.-T. Kim, S.-K. Chung MedChem Comm 2013, 4, 310.

24 C. V. Bonduelle, E. R. Gillies Pharmaceuticals 2010, 3, 636.

25 A. Battigelli, J. T.-W. Wang, J. Russier, T. Da Ros, K. Kostarelos, K. Y. Al-Jamal, M. Prato, A. Bianco Small 2013, 9, 3610.

26 V. Bagnacani, V. Franceschi, M. Bassi, M Lomazzi, G. Donofrio, F. Sansone, A. Casnati, R. Ungaro Nat. Commun. 2013, 4, 1721.

27 J. Gasparello, A. Manicardi, A. Casnati, R. Corradini, R. Gambari, A. Finotti, F. Sansone Scientific Report 2019, 9, 3036.

28 J. Fernández-Carneado, M. Van Gool, V. Martos, S. Castel, P. Prados, J. de Mendoza, E. Giralt J. Am. Chem. Soc. 2005, 127, 869.

29 T. J. Baker, N. W. Luedke, Y. Tor, M. Goodman J. Org. Chem. 2000, 65, 9054.

30 N. W. Luedke, P. Carmichael, Y. Tor J. Am. Chem. Soc. 2003, 125, 12374.

31 L. Elson-Schwab, O. B. Garner, M. Schuksz, B. E. Crawford, J. D. Esko, Y. Tor J. Biol. Chem. 2007, 282, 13585.

32 A. V. Dix, L. Fischer, S. Sarrazin, C. P. Redgate, J. D. Esko, Y. Tor ChemBioChem 2010, 11, 2302.

33 M. Inoue, W. Tong, J. D. Esko, Y. Tor ACS Chem. Biol. 2013, 8, 1383.

34 S. Sarrazin, B. Wilson, W. S. Sly, Y. Tor, J. D. Esko Mol. Ther. 2010, 18, 1268.

35 W. Tong, C. A. Dwyer, B. E. Thacker, C. A. Glass, J. R. Brown, K. M. Hamill, K. W. Moremen, S. Sarrazin, P. L. S. M. Gordts, L. E. Dozier, G. N. Patrick, Y. Yor, J. D. Esko Mol. Ther. 2017, 25, 2743.

36 E. Wexselblatt, J. D. Esko, Y. Tor ACS Nano 2015, 9, 3961.

37 K. M. Hamill, E. Wexselblatt, W. Tong, J. D. Esko, Y. Tor J. Mater. Chem. B 2016, 4, 5794. 
38 A. Ghilardi, D. Pezzoli, M. C. Bellucci, C. Malloggi, A. Negri, A. Sganappa, G. Tedeschi, G. Candiani, A. Volonterio Bioconjugate Chem. 2013, 24, 1928.

39 A. Sganappa, E. Wexselblatt, M. C. Bellucci, J. D. Esko, G. Tedeschi, Y. Tor, A. Volonterio ChemBioChem, 2017, 18, 119.

40 N. Bono, C. Pennetta, M. C. Bellucci, A. Sganappa, C. Malloggi, G. Tedeschi, G. Candiani, A. Volonterio ACS Omega 2019, 4, 6796.

41 N. Bono, C. Pennetta, A. Sganappa, E. Giupponi, F. Sansone, A. Volonterio, G. Candiani Int. J. Pharm. 2018, 549, 436

42 J. M. J. M. Ravasco, H. Faustino, A. Trindade, P. M. P. Gois Chem. Eur. J. 2019, 25, 43.

43 K. Renault, J. W. Fredy, P.-Y. Renard, C. Sabot Bioconjugate Chem. 2018, 29, 2497.

44 The stability of the thiol-maleimide product has been recently questioned due to the possible occurring slow retro-Michael process. However, hydrolysis of the maleimide ring blocks the reversibility of the thio-Michael addition resulting in a stable succinimide acid adduct: N. L. Tumey, M. Charati, T. He, E. Sousa, D. Ma, X. Han, T. Clark, J. Casavant, F. Loganzo, F. Barletta, J. Lucas, E. I. Graziani Bioconjugate Chem. 2014, 25, 1871.

45 A. Beck, L. Goetsch, D. Dumontet, N. Corvaïa Nat. Rev. Drug Discovery 2018, 16, 315.

46 O. Koniev, A. Wagner Chem. Rev. Soc. 2015, 44, 5495.

47 Compound 3 was prepared as described in: J. Han, L. Sun, Y. Chu, Z. Li, D. Huang, X. Zhu, H. Qian, W. Huang J. Med. Chem. 2013, 56, 9955.

48 Compound 4 was prepared as described in: M. V. Spanedda, L. Bourel-Bonnet Bioconjugate Chem. 2021, 32, 482.

49 Compound 8 was prepared as described in: A. Natarajan, W. Du, C.-Y. Xiong, G. L. DeNardo, S. J. DeNardo, J. Gervay-Hague Chem. Commun. 2007, 7, 695.

50 B.-Q. Shen, K. Xu, L. Liu, H. Raab, S. Bhakta, M. Kenrick, K. L. Parsons-Reponte, J. Tien, S.-F. Yu, E. Mai, D. Li, J. Tibbitts, J.

Baudys, O. M. Saad, S. J. Scales, P. J. McDonald, P. E. Hass, C. Eigenbrot, T. Nguyen, W. A. Solis, R. N. Fuji, K. M. Flagella, D. Patel, S. D. Spencer, L. A. Khawli, A. Ebens, W. L. Wong, R. Vandlen, S. Kaur, M. X. Sliwkowski, R. H. Scheller, P. Polakis, J. R. Junutula Nat. Biotechnol. 2012, 30, 184.

51 M. A. Krzyzaniak, M.T. Zumstein, J. A. Gerez, P. Picotti, A. Helenius PLOS Pathogens, 2013, 9, e1003309.

52 C. C. Norbury Immunology 2006, 117, 44.

53 A. K. Edwards, S. Ramesh, V. Singh, C. Tayade Mol. Hum. Reprod. 2014, 20, 1002.

54 A. Gupta, S. Inaba, O. K. Wong, G. Fang, J. Liu Oncogene 22 7593.

55 S. Inaba, C. Li, E. Shi, D.-Q. Song, J.-D. Jiang, J. Liu Breast Cancer Res. Treat. 2005, 94, 25.

56 L. Wei, X.-W. Liang, Q.-H. Zhang, M. Li, J. Yuan, S. Li, S.-C. Sun, Y.-C. Ouyang, H. Schatten, Q.-Y. Sun Cell Cycle 2010, 9, 1112.

57 M. Malumbres, M. Barbacid Curr. Opin. Genet. Dev. 2007, 17, 60.

58 R. R. Traut, A. Bollen, T. T. Sun, J. W. Hershey, J. Sundberg, L. R. Pierce Biochemistry 1973, 12, 3266.

59 I. M. Klotz, R. E. Heiney Arch. Biochem. Biophys. 1962, 96, 605.

60 Y. Zhou, G. Wang, P. Wang, Z. Li, T. Yue, J. Wang, P. Zou Angew. Chem. Int. Ed. 2019, 58, 11763. 